\title{
Three-Dimensional Numerical Investigation of an Improved Surface Plasmon Resonance Waveguide Sensor
}

\author{
Jun Shibayama, Member, IEEE
}

\begin{abstract}
A light-guiding structure is investigated to improve the performance of a surface plasmon resonance waveguide sensor using the three-dimensional beam-propagation method. The absorption strength at the resonance wavelength is examined in terms of the core width. Calculations show that a waveguide with a wide core supporting higher order modes leads to strong absorption comparable to the two-dimensional model. Besides, embedding the core in a substrate is quite effective in obtaining a sharp dip in the wavelength response, i.e., a full-width at half-minimum is reduced from $\simeq 11$ to $\simeq 5 \mathrm{~nm}$, when the core is embedded by $0.3 \mu \mathrm{m}$. This contributes to improved measurement precision.
\end{abstract}

Index Terms-Beam-propagation method (BPM), optical waveguide, surface plasmon resonance (SPR) sensor, waveguide-based sensor.

\section{INTRODUCTION}

A SURFACE plasmon resonance (SPR) sensor consisting of an optical waveguide, compatible with conventional planar waveguide technology, has received much attention due to its advantages such as small size, ruggedness, and multichannel sensing. In previous works, the theoretical investigations of SPR waveguide sensors were limited to two-dimensional (2-D) models [1]-[5]. Note that the 2-D models cannot fully predict the characteristics of practical three-dimensional (3-D) models of the SPR sensors, since the transverse dimensions are not taken into account. Hence, we have applied the 3-D beam-propagation method (BPM) to the evaluation of the basic characteristics of a 3-D model [6]. Unfortunately, the absorption strength at the resonance wavelength for the 3-D model consisting of a single-mode waveguide has been found to be less than the corresponding 2-D counterpart [6]. This is qualitatively consistent with the relation between the measured result of a single-mode waveguide sensor and the 2-D calculated one [2]. We are, therefore, motivated to study a practical 3-D sensor structure possessing not only strong absorption but also a sharp dip in the wavelength response, with a concomitant improvement of measurement precision.

In this letter, we investigate a light-guiding structure for improved performance of an SPR waveguide sensor using the 3-D

Manuscript received January 21, 2010; revised February 08, 2010; accepted February 09, 2010. Date of publication February 22, 2010; date of current version April 09, 2010. This work was supported in part by MEXT, Grant-in-Aid for Young Scientists (B) (21760266).

The author is with the Faculty of Engineering, Hosei University, Tokyo 1848584, Japan (e-mail: shiba@hosei.ac.jp).

Digital Object Identifier 10.1109/LPT.2010.2043428

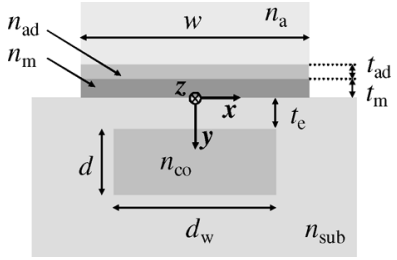

Fig. 1. Cross section of an SPR waveguide sensor.

semivectorial BPM. First, we examine the absorption strength, paying attention to the core width. It is shown that a waveguide with a wide core supporting higher order modes leads to the strong absorption comparable to that of the 2-D model. Next, we discuss the effect of an embedded core on the wavelength response. Calculations show that the embedded core is effective in achieving a sharp dip in the wavelength response, i.e., a full-width at half-minimum (FWHM) is appreciably reduced from $\simeq 11$ to $\simeq 5 \mathrm{~nm}$, when the core is embedded by $0.3 \mu \mathrm{m}$, while maintaining the sensitivity to the refractive index change of the analyte.

\section{DISCUSSION}

Fig. 1 illustrates the cross section of the SPR waveguide sensor to be studied. The adsorbed layer (or tuning layer) [2] is inserted between the metal and analyte layers for the sensor operation around $\lambda=0.6 \mu \mathrm{m}$, where these layers are centered on the waveguide $\left(t_{m}=0.045 \mu \mathrm{m}\right.$ and $t_{\mathrm{ad}}=0.02 \mu \mathrm{m}$ [6]). The sensing length is $z=200 \mu \mathrm{m}$. The refractive indices are chosen to be $n_{\mathrm{co}}=n_{\mathrm{ad}}=1.47$ and $n_{\mathrm{sub}}=1.46$. The metal is selected to be $\mathrm{Au}\left(n_{m}=0.131-j 3.654\right.$ at $\left.0.6328 \mu \mathrm{m}\right)$ and the dispersion property of its refractive index is taken into account using the Drude model [6]. We excite the dielectric transverse-magnetic (TM) mode (without the metal, adsorbed, and analyte layers) as an input field. Varying the operating wavelength, we evaluate the output power from the waveguide, which depends on the refractive index of an analyte $n_{a}$. Water is used as the analyte, which is sufficiently thick $(1 \mu \mathrm{m})$ to yield a converged solution.

We employ the BPM based on the alternating-direction implicit scheme [6], [7]. To remove numerical instability often encountered in the vicinity of metal, we adopt the complex reference refractive index approach [8] equivalent to the branch-cut rotation method [9]. In particular, we apply the complex reference index only to the metal region where the reference index $n_{0}$ in the coefficient of the first $z$ derivative in the Fresnel equation 


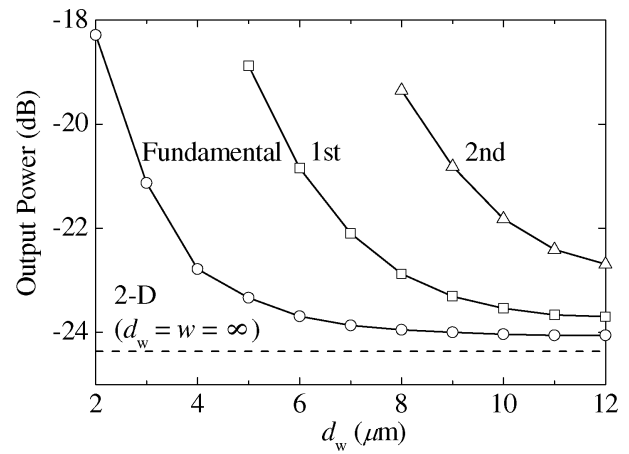

Fig. 2. Minimum output power at the resonance wavelength versus core width $\left(t_{e}=0\right)$.

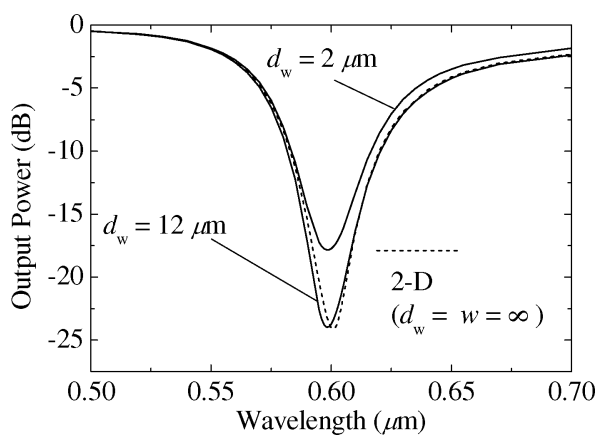

Fig. 3. Wavelength responses obtained from the fundamental mode excitation.

is changed into a complex value, while $n_{0}$ in the phase variation term $k^{2}\left(n^{2}-n_{0}^{2}\right)$ is kept real [10] ( $k$ is the free space wavenumber and $n$ is the refractive index of the structure). In what follows, we use $n_{0}=\bar{n}_{0} e^{i \rho}$ with $\bar{n}_{0}$ and $\rho$ being the effective index of the dielectric TM mode and $-5^{\circ}$, respectively $\left(\bar{n}_{0}\right.$ is the original real reference index and $\rho$ is a phase factor). The structure is discretized with $\Delta x=0.05 \mu \mathrm{m}, \Delta y=0.005 \mu \mathrm{m}$, and $\Delta z=0.1 \mu \mathrm{m}$.

First, we examine the dependence of the core width $d_{w}$ on the absorption strength, which cannot be treated in the 2-D models [1]-[5], [11], [12]. The metal strip is wide enough to cover the core region $(w=20 \mu \mathrm{m})$. The core thickness is deliberately chosen to be $d=2 \mu \mathrm{m}$ throughout this letter so that the singlemode operation in the $y$ direction may be maintained. This is due to the fact that a waveguide with a thick core supporting higher order modes in the $y$ direction results in a broadening of the absorption dip in the wavelength response, when compared with the single-mode case [12].

Fig. 2 shows the minimum output power at the resonance wavelength with varying the core width $d_{w}$ for $t_{e}=0$ (semi-embedded core) and $n_{a}=1.332$, along with the result of the 2-D model $\left(d_{w}=w=\infty\right)$. As is observed, the resonances for the first and second modes in the transverse direction start to appear around $d_{w}=5$ and $8 \mu \mathrm{m}$, respectively. The output power for each mode gradually approaches the 2-D result, as the core width becomes wide. Here we check the wavelength response obtained from the fundamental mode excitation. Fig. 3 depicts the SPR responses for $d_{w}=2 \mu \mathrm{m}$ (single-mode waveguide) and

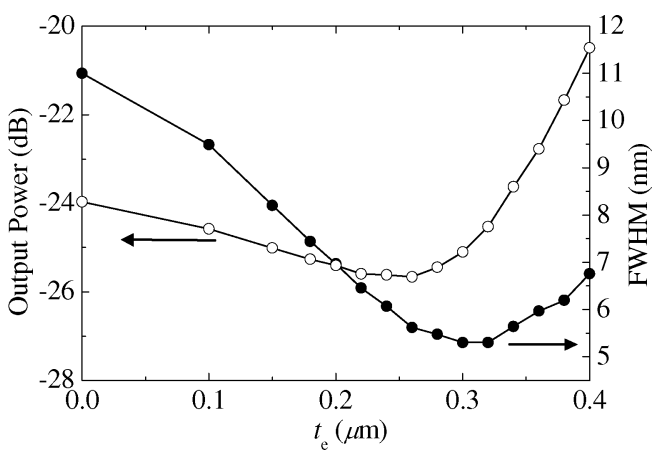

Fig. 4. Minimum output power at the resonance wavelength and FWHM of the wavelength response versus $t_{e}$.

$12 \mu \mathrm{m}$. For reference, the response of the 2-D model is also included with the dashed line. As expected from Fig. 2, the absorption for $d_{w}=2 \mu \mathrm{m}$ is much weaker than that for $d_{w}=12 \mu \mathrm{m}$, giving rise to less measurement sensitivity. It should be noted that the response for $d_{w}=12 \mu \mathrm{m}$ is almost the same as the 2-D counterpart. Consequently, the core width should be chosen wide, in order to achieve the wavelength responses predicted by the 2-D model.

We now briefly mention the characteristics obtained from the higher order mode excitation. For a specific $d_{w}$, the lower absorption of the higher order modes found in Fig. 2 stems from the extension of the higher order mode field compared with the fundamental mode field. We also calculate the wavelength responses of the first and second modes for $d_{w}=12 \mu \mathrm{m}$. As a result, almost the same wavelength responses as the response of the fundamental mode are obtained, although not illustrated in Fig. 3. This indicates that the strong absorption can be maintained, even when several modes are simultaneously entered into the sensing section.

Next, we discuss the effect of the embedded core on the absorption strength. Note that its effect is similar to that of a buffer layer placed between the core and the metal layer [3]-[5]. Again, theoretical investigations on this subject have been restricted to 2-D models. Although the embedded core was used for the sensor with the ion-exchanged graded-index waveguide in [13], its effect was not presented explicitly. Here, we treat a full 3-D structure with a core width of $d_{w}=12 \mu \mathrm{m}$.

Fig. 4 represents the minimum output power at the resonance wavelength and the FWHM of the wavelength response versus $t_{e}$ for the fundamental mode excitation. It is apparent that the output power and the FWHM exhibit local minimum values around $t_{e}=0.3 \mu \mathrm{m}$. In particular, the FWHM of the response is appreciably reduced to $\simeq 5 \mathrm{~nm}$ for $t_{e}=0.3 \mu \mathrm{m}$, demonstrating the effectiveness of the embedded core. Fig. 5 displays the wavelength responses for $t_{e}=0$ and $3 \mu \mathrm{m}\left(d_{w}=12 \mu \mathrm{m}\right)$. The response is found to become sharper for $t_{e}=0.3 \mu \mathrm{m}$, leading to the improved measurement precision. Note even with the embedded core that the sensitivity to the refractive index change of the analyte $n_{a}$ is successfully maintained: the absorption wavelength shifts from 0.594 to $0.602 \mu \mathrm{m}$, when the refractive index of the analyte is increased from 1.330 to 1.334 [6].

Finally, we clarify the reason why the embedded core contributes to a sharp dip in the wavelength response. To do so, 


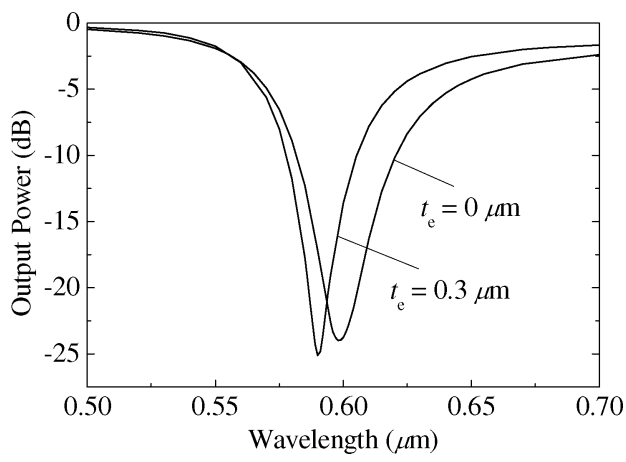

Fig. 5. Wavelength responses obtained from the fundamental mode excitation $\left(d_{w}=12 \mu \mathrm{m}\right)$.

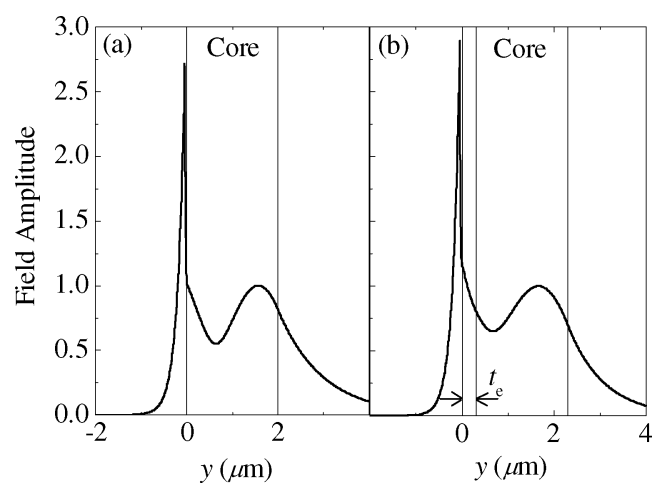

Fig. 6. Eigenmode field $\left(H_{x}\right)$ at the resonance wavelength. (a) $t_{e}=0$ and (b) $t_{e}=0.3 \mu \mathrm{m}$.

we perform the 2-D eigenmode analysis of the sensor using the imaginary-distance BPM [7]. Note that there exist two eigenmodes at the resonance wavelength in the sensor section [6]. The higher modes, for example, are illustrated in Fig. 6 for (a) $t_{e}=0$ and (b) $t_{e}=0.3 \mu \mathrm{m}$, in which each field is normalized to its maximum value in the core region. The surface plasmon wave can be seen around the interface between the metal and the analyte. Close observation reveals that the amplitude of the surface plasmon wave for $t_{e}=0.3 \mu \mathrm{m}$ is larger than that for $t_{e}=0$ (this is also true for the lower mode cases). That is, the SPR becomes strong with the embedded core being used, leading to the significant field absorption in the metal. As a result of the strong resonance, the wavelength response becomes sharper, as shown in Fig. 5.

\section{CONCLUSION}

We have investigated an improved SPR waveguide sensor using the 3-D BPM. Numerical results suggest that the 3-D analysis is indispensable for evaluating the practical model of the SPR waveguide sensor. It is found that the waveguide supporting higher order modes in the transverse direction attains strong absorption comparable to the 2-D model; in other words, the SPR sensor consisting of a single-mode waveguide cannot achieve the wavelength response predicted by the frequently used 2-D techniques. Besides, the waveguide with the embedded core is shown to be effective in further reducing the output power at the resonance wavelength. The reduction leads to a sharp dip in the wavelength response, which contributes to the improved measurement precision. Application to the absorption-based waveguide sensor [14] is left for a future study.

\section{ACKNOWLEDGMENT}

The author would like to thank Prof. J. Yamauchi and Prof. H. Nakano of Hosei University for their encouragement in his work. The author also thanks Assoc. Prof. M. Fujimura of Osaka University for sending reference [13] and giving a chance to investigate the effect of an embedded core of an SPR waveguide sensor.

\section{REFERENCES}

[1] J. Čtyroký, J. Homola, P. V. Lambeck, S. Musa, H. J. W. M. Hoekstra, R. D. Harris, J. S. Wilkinson, B. Usievich, and N. M. Lyndin, "Theory and modelling of optical waveguide sensors utilising surface plasmon resonance," Sens. Actuators B, vol. 54, no. 1-2, pp. 66-73, Jan. 1999.

[2] J. Čtyroký, J. Homola, and M. Skalsky, "Tuning of spectral operation range of a waveguide surface plasmon resonance sensor," Electron. Lett., vol. 33, no. 14, pp. 1246-1248, Jul. 1997.

[3] C. R. Lavers and J. S. Wilkinson, "A wave-guide-coupled surfaceplasmon sensor for an aqueous environment," Sens. Actuators B, vol. 22, no. 1, pp. 75-81, Oct. 1994.

[4] R. D. Harris and J. S. Wilkinson, "Waveguide surface plasmon resonance sensors," Sens. Actuators B, vol. 29, no. 1-3, pp. 261-267, Oct. 1995.

[5] M. N. Weiss, R. Srivastava, and H. Groger, "Experimental investigation of a surface plasmon-based integrated-optic humidity sensor," Electron. Lett., vol. 32, no. 9, pp. 842-843, Apr. 1996.

[6] J. Shibayama, S. Takagi, T. Yamazaki, J. Yamauchi, and H. Nakano, "Numerical analysis of waveguide-based surface plasmon resonance sensor with adsorbed layer using two- and three-dimensional beampropagation methods," IEICE Trans. Electron., vol. E90-C, no. 1, pp. 95-101, Jan. 2007.

[7] J. Shibayama, T. Yamazaki, J. Yamauchi, and H. Nakano, "Eigenmode analysis of a light-guiding metal line loaded on a dielectric substrate using the imaginary-distance beam-propagation method," J. Lightw. Technol., vol. 23, no. 3, pp. 1533-1539, Mar. 2005.

[8] H. L. Rao, M. J. Steel, R. Scarmozzino, and R. M. Osgood Jr., "Complex propagators for evanescent waves in bidirectional beam propagation method," J. Lightw. Technol., vol. 18, no. 8, pp. 1155-1160, Aug. 2000.

[9] H. El-Refaei, I. Betty, and D. Yevick, "The application of complex Padé approximants to reflection at optical waveguide facets," IEEE Photon. Technol. Lett., vol. 12, no. 2, pp. 158-160, Feb. 2000.

[10] J. Shibayama, T. Takeuchi, N. Goto, J. Yamauchi, and H. Nakano, "Numerical investigation of a Kretschmann-type surface plasmon resonance waveguide sensor," J. Lightw. Technol., vol. 25, no. 9, pp. 2605-2611, Sep. 2007

[11] P. Stocker, B. Menges, U. Langbein, and S. Mittler, "Multimode waveguide mode surface plasmon coupling: a sensitivity and device realizability study," Sens. Actuators A, vol. 116, no. 2, pp. 224-231, Oct. 2004.

[12] Y. S. Chu, W. H. Hsu, C. W. Lin, and W. S. Wang, "Surface plasmon resonance sensors using silica-on-silicon optical waveguides," Microw. Opt. Technol. Lett., vol. 48, no. 5, pp. 955-957, May 2006.

[13] T. Harada, T. Takiguchi, and K. Hakamata, "Sensitivity improvement of optical waveguide type SPR sensors," in 3rd Int. Nanophotonics Symp., Handai, Japan, Jul. 2006, Paper P-40.

[14] J. Shibayama, "A Kretschmann-type absorption-based surface plasmon resonance waveguide sensor," Microw. Opt. Technol. Lett., vol. 50, no. 10, pp. 2497-2500, Oct. 2008. 